\title{
Facile Fabrication of Breathable and Superhydrophobic Fabric based on Silica Nanoparticles and Amino-Modified Polydimethylsiloxane
}

\author{
Mahdi Hasanzadeh ${ }^{1 *}$, Hossein Shahriyari Far ${ }^{2}$, Aminoddin Haji ${ }^{1}$, Giuseppe Rosace ${ }^{3}$ \\ ${ }^{1}$ Department of Textile Engineering, Yazd University, P.O. Box 89195-741, Yazd, Iran \\ ${ }^{2}$ Department of Chemistry, Iran University of Science and Technology, Narmak, Tehran, Iran \\ ${ }^{3}$ Department of Engineering and Applied Sciences, University of Bergamo, Viale Marconi 5, \\ 24044, Dalmine, BG, Italy \\ *Corresponding Author: m.hasanzadeh@yazd.ac.ir - Fax: +98-353-8209817; Tel: +98-353- \\ 1232569
}

\begin{abstract}
This work attempted to fabricate superhydrophobic fabric via simple immersion technique. Textile fabrics were coated with silica nanoparticles prepared from tetraethoxysilane (TEOS) to obtain sufficient roughness with hydrophobic surface chemistry. Then the coated fabrics were treated with polydimethylsiloxane (PDMS) and aminopropyltriethoxysilane (APTES) to reduce the surface energy. The effects of PDMS concentration on the surface morphology and superhydrophobicity of as-prepared fabric were investigated. The morphology and the composition of superhydrophobic fabric was characterized by scanning electron microscopy (SEM), energy dispersive X-ray (EDS) and Fourier transform infrared (FTIR) spectroscopy. The results revealed the formation of spherical silica nanoparticles with average particle size of 250 $\mathrm{nm}$ throughout the fabric surface. The possible interactions between silica nanoparticles and APTES, as well as the fabrics were elucidated. Investigating the hydrophobicity of fabrics via water contact angle (WCA) measurement showed that the treated fabric exhibits excellent water repellency with a water contact angle as high as $151^{\circ}$ and a very low water sliding angle. It also found that the treated fabric maintained most of its hydrophobicity against repeated washing. The comfort properties of the obtained superhydrophobic fabrics in term of air permeability and bending length did not reveal any significant changes.
\end{abstract}


Keywords: Sol-gel; Hybrid coating; Superhydrophobic; textile fabric; polydimethylsiloxane; contact angle

\section{Introduction}

A surface with a water contact angle (at equilibrium) higher than $150^{\circ}$ and contact angle hysteresis lower than $10^{\circ}$ is considered as a superhydrophobic surface. Nowadays superhydrophobic coatings have gained increasing attention due to their diverse applications including water repellent and self-cleaning textiles, anti-icing surfaces suitable for power network equipment, anticorrosion devices, biomedical devices, construction industry, antibacterial fabrics, anti-biofouling surfaces in marine industry, and oil-water separation [1]-[3].

A combination of micro-nano roughness and low surface energy can lead to a superhydrophobic surface. So, an intrinsically hydrophobic substrate can be converted to a superhydrophobic structure by creating micro-nano roughness on its surface by means of plasma etching, chemical etching, nanoparticle attachment, etc. The hydrophilic surfaces, superhydropobicity can be obtained by chemically modifying the micro-nano rough surface with low surface energy compounds [2], [4], [5].

One of the widely studied strategies for fabrication of superhydrophobic textiles is based on increasing the surface roughness by coating of the surface with inorganic nanoparticles through the sol-gel process and subsequently lowering the surface energy by attachment of a hydrophobic compound on its surface. Also, using a sol, modified with an appropriate hydrophobic compound, may lead to a superhydrophobic surface coating by a one step process [6]. Hoefnagels et al. [7] turned the hydrophilic cotton to superhydrophobic by a two-step process including the sol-gel based in-situ growing of silica micro-particles on cotton fibers followed by a hydrophobization step using polydimethylsiloxane (PDMS). Xu et al. [8] examined the coating of cotton surface with $\mathrm{SiO}_{2}$ nanoparticles or $\mathrm{ZnO}$ nanorods for creation of nano-roughness and modification of the rough surface with n-dodecyltrimethoxysilane (DTMS) to lower the surface energy and prepared superhydrophobic cotton fabrics. Based on their results, cotton fabrics prepared based on $\mathrm{SiO}_{2}$ nanoparticles and $\mathrm{ZnO}$ nanorods showed static water contacts angles (WCAs) of $159^{\circ}$ and $153^{\circ}$ respectively. 
Superhydrophobic PET fabric was obtained by a two-step process including coating with $\mathrm{SiO}_{2}$ nanoparticles and PDMS in the first step and subsequent hydrophobization through a sol-gel process using tetraethoxysilane and cetyltrimethoxysilane. The coated textile showed a WCA of $162.5^{\circ}$ and was resistant to hydrostatic pressure up to $38.6 \mathrm{KPa}$ [9]. Xue et al. [10] fabricated a self-healing and superhydrophobic PET fabric using polydimethylsiloxane and octadecylamine (ODA) through a dip-coating followed by curing procedure. The required roughness was provided by the self- roughed property of ODA and the obtained superhydropobicity was durable to abrasion (5000 cycles), and washing (120 cycles). $\mathrm{TiO}_{2}$ sol modified with poly(hexafluorobutyl methacrylate) was another approach employed by Yang et al. [11] to prepare superhydrophobic cotton fabrics with WCA of $152.5^{\circ}$. Chauhan et al. reported the preparation of superhydrophobic cotton with self-cleaning and stain resistant properties by simple coating with hexadecyltrimethoxysilane by immersion-drying method. WCA of $157^{\circ}$ was obtained. It was concluded that the high contact angle is due to the hierarchical microstructures and presence of long-chain alkyl groups on the modified cotton surface [12].

Herein, we present facile and non-fluorinated approach to construct superhydrophobic and breathable fabric based on hydrolysis and condensation of tetraethylorthosilicate (TEOS) followed by crosslinking with amino-modified polydimethylsiloxane (PDMS). The formation of silica nanoparticles, morphology and structure of treated fabric were investigated by scanning electron microscopy (SEM), energy dispersive X-ray (EDS) and Fourier transform infrared (FTIR) spectroscopy. The treated fabric exhibited excellent eater repellency with durable washing fastness.

\section{Experimental procedure}

\subsection{Materials}

Poly(dimethylsiloxane) mono-glycidyl ether terminated (PDMS) and tetraethylorthosilicate (TEOS, 98\%) were purchased from Sigma-Aldrich. Ammonium hydroxide (25\% in water), ethanol $\left(\mathrm{C}_{2} \mathrm{H}_{6} \mathrm{O}\right)$, toluene $\left(\mathrm{C}_{7} \mathrm{H}_{8}\right)$ and 3-aminopropyltriethoxysilane (APTES) were supplied from Merck Co. (Germany), and used without additional purification. Commercial polyester-viscose fabrics $\left(350 \mathrm{~g} / \mathrm{m}^{2}\right)$ was used as the substrate. The fabric was rinsed with detergent and distilled water separately several times and dried at $100{ }^{\circ} \mathrm{C}$ for $30 \mathrm{~min}$. Deionized water was used in all the prepared solutions.

\subsection{Fabrication of silica-coated fabrics}


The spherical silica nanoparticles were prepared by the modified Stober method [13]. Briefly, first, a mixture of TEOS $(0.39 \mathrm{~mol})$ and ethanol $(2.35 \mathrm{~mol})$ was prepared as a solution A. Similarly, $2.94 \mathrm{~mol}$ distilled water, $2.35 \mathrm{~mol}$ ethanol, and $\mathrm{NH}_{4} \mathrm{OH}$ were mixed to form solution B. Then, the solutions A and B were mixed together at $40^{\circ} \mathrm{C}$ for $40 \mathrm{~min}$. Finally, the washed fabric immersed in a solution of silica nanoparticles for $20 \mathrm{~min}$ and after dip-coating, the fabrics were left at room temperature to remove the solvent and cured at $80{ }^{\circ} \mathrm{C}$ for $10 \mathrm{~min}$.

\subsection{Fabrication of superhydrophobic fabric}

In this procedure, different amounts of PDMS were first added into $50 \mathrm{~mL}$ toluene to find the optimum value. After stirring about $60 \mathrm{~min}, 0.5 \mathrm{~mL}$ APTES solution was added. Then, the mixture was stirred $(500 \mathrm{rpm})$ for $3 \mathrm{~h}$ at room temperature. Finally, the silica-coated fabrics were dip-coated in APTES-PDMS solution for $20 \mathrm{~min}$ and cured at $120^{\circ} \mathrm{C}$ for $60 \mathrm{~min}$. The schematic representation of the superhydrophobic coating of fabric is shown in Figure 1.

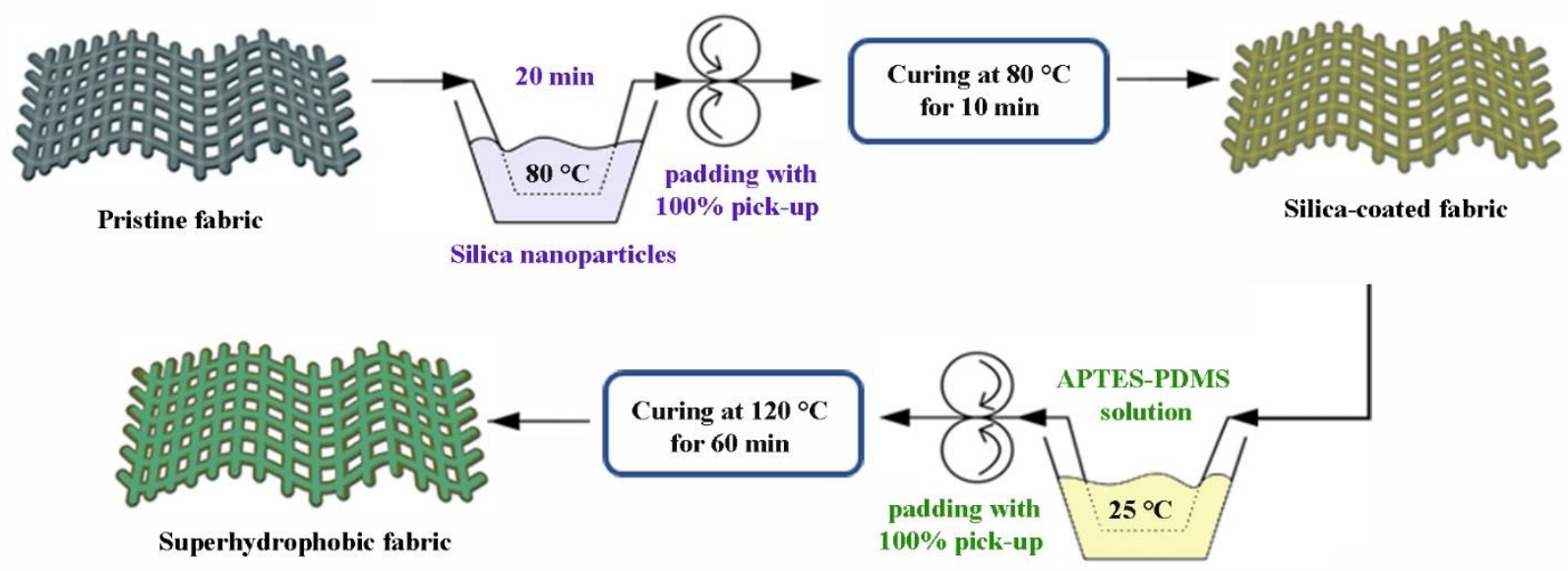

Figure 1. Schematic representation of fabric superhydrophobic coating.

\subsection{Characterization}

The surface morphology of pristine and treated fabrics as well as the composition of materials were studied after gold coating of samples by scanning electron microscopy (Hitachi su3500) and energy-dispersive X-ray spectroscopy (EDS). Fourier transformed infrared (FTIR) spectra of samples were recorded on AVATAR FTIR instrument (Thermo Nicolet, USA) to study the functional group analysis and possible reactions. 
Water contact angle (CA) measurement was carried out at room temperature using home-made instrumentation including microscope equipped with a CCD camera and PCTV vision software. A $5 \mu \mathrm{L}$ water droplet was dropped to the five different location of fabric surface and average value was reported. Wash fastness of superhydrophobic fabric, as an important characteristic in terms of users, was evaluated based on AATCC Test Method 61-2A. The treated fabrics were washed with aqueous solution of anionic detergent $(0.23 \%)$ followed by rinsing with water and drying at $49{ }^{\circ} \mathrm{C}$ for $45 \mathrm{~min}$, after each washing cycle. According to this standard, each washing cycle is equivalent to five home machine laundering at $38^{\circ} \mathrm{C}$. The washing operation was repeated for five times. Air permeability of treated samples were evaluated based on ASTM-D737-75 by using SDL-Air Permeability device. Crease recovery angle was measured based on ASTMD 123 standard with Shrirley device. Bending test, as very important factor reflecting the flexibility of the fabrics, was performed according to ASTM-D1388 standard with Shirley Bending length device. Five samples (both warp and weft) were measured, and the average value was reported.

\section{Results and discussion}

\subsection{Morphological analysis}

Figure 2 shows the SEM micrographs of pristine and treated fabrics. The results exhibited a rough surface and the presence of silica nanoparticles on the surface of fabric. The high-magnification (inset) SEM image (Figure 2b) showed that the uniform silica nanoparticles deposited throughout the fabric surface with average particle size of about $290 \mathrm{~nm}$. After modification of silica-coated fabric with PDMS-APTES solution, the surface morphology becomes inhomogeneous with sticky particle. The change in morphology of PDMS-APTES treated silica-coated fabric is clearly shown in Figure 2c. The results of EDS spectra (Figure 2d) confirm the presence of the Si element on the superhydrophobic fabric surface. The peaks of $\mathrm{C}$ and $\mathrm{O}$ elements were also detected in EDS spectra which are attributed to the polymer chain. 

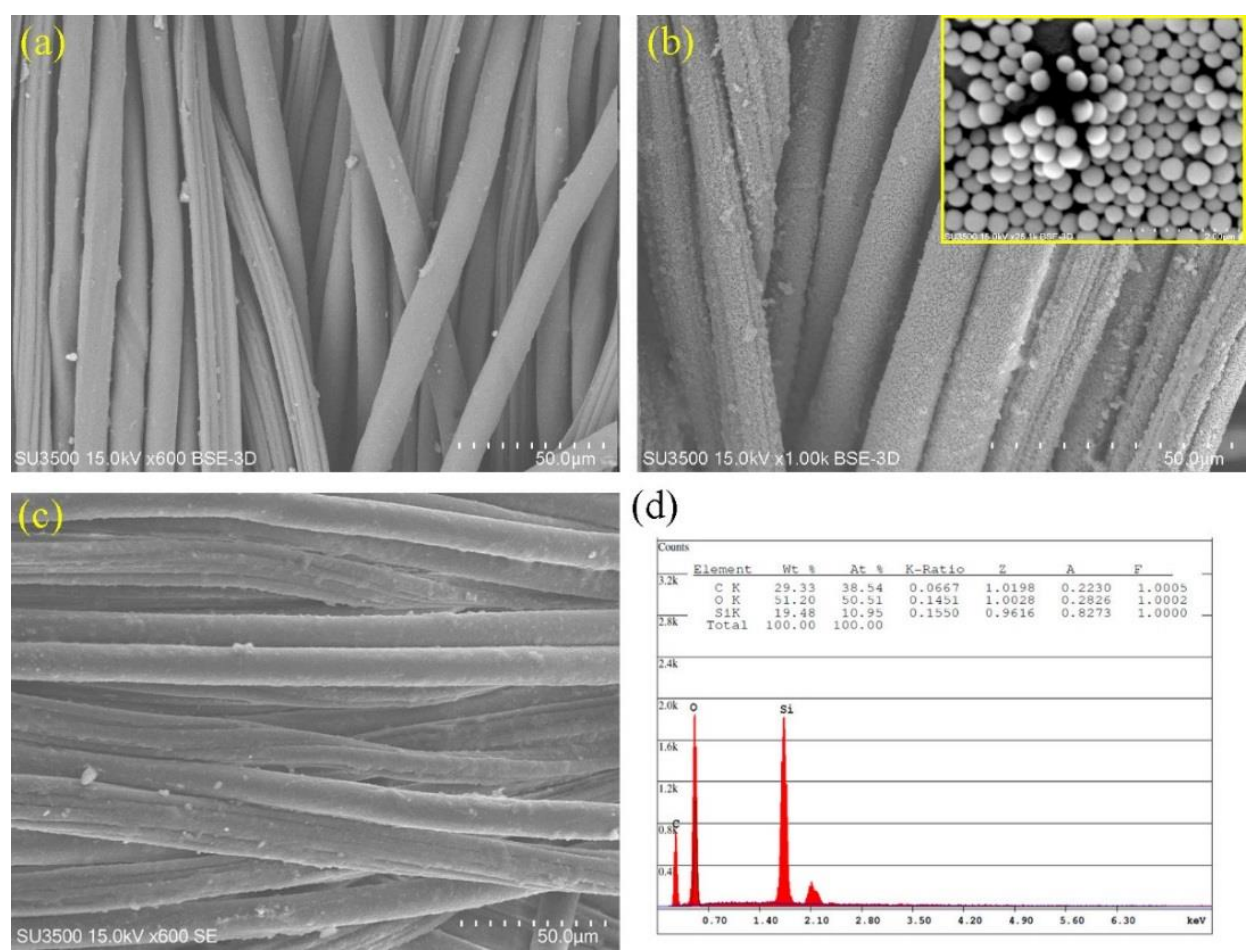

Figure 2. SEM micrographs of fabrics: (a) pristine, (b) silica-coated, (c) PDMS-APTES treated, and (d) EDS pattern of superhydrophobic fabric.

\subsection{Structural analysis}

Amino-modification of mono-glycidyl ether terminated PDMS with APTES has been carried out and the possible reactions was investigated by FTIR (Figure 2a). Diminishing the characteristic absorption band of epoxide ring at $913 \mathrm{~cm}^{-1}$ as well as increasing the hydroxyl and amine groups at 3420 and $1495 \mathrm{~cm}^{-1}$, respectively, confirm the successful amine-modification of PDMS [14]. As can be seen in spectrum of modified PDMS, the absorption band of oxirane group was completely disappeared, revealing complete reaction between APTES and PDMS. The possible chemical reaction process between silica nanoparticles and modified PDMS is represented in Scheme 1. The FTIR spectrum of pristine and treated fabrics are shown in Figure 2b. As can be seen, the Si-O-Si absorption band near $1100 \mathrm{~cm}^{-1}$ becomes sharper and more intense after treatment of fabric with silica and modified PDMS. 

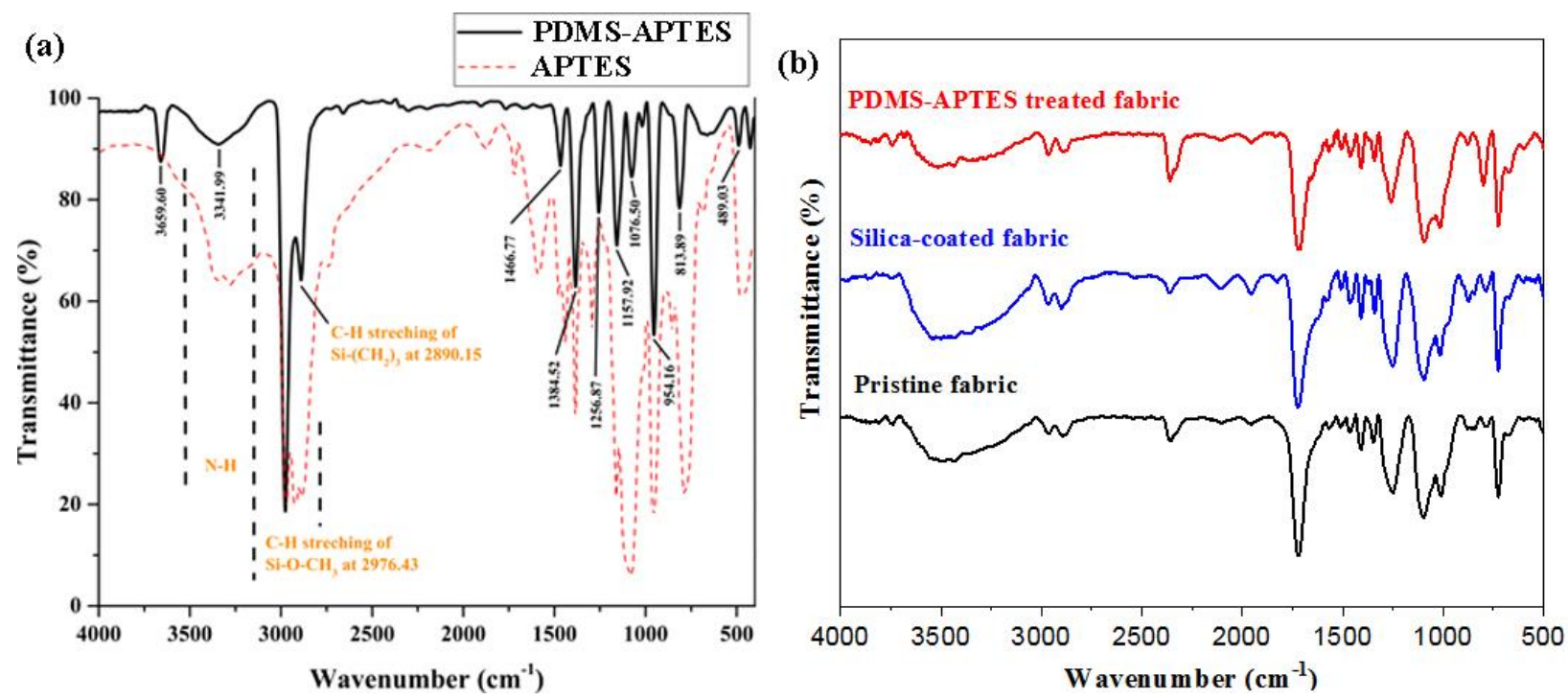

Figure 3. FTIR spectra of (a) amino-modified PDMS solution, (b) pristine and treated fabrics.

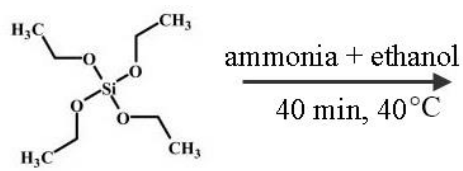

TEOS

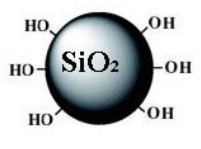

(I)<smiles>CCCOC(CCC)(CCC)CCC</smiles>

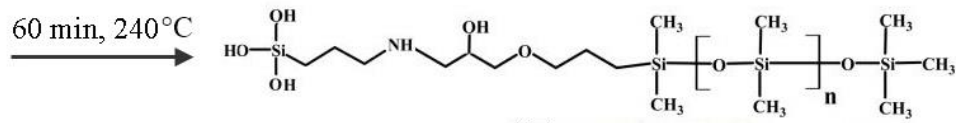<smiles>CC(C)(C)CCCOCCC1CO1</smiles>

(II)

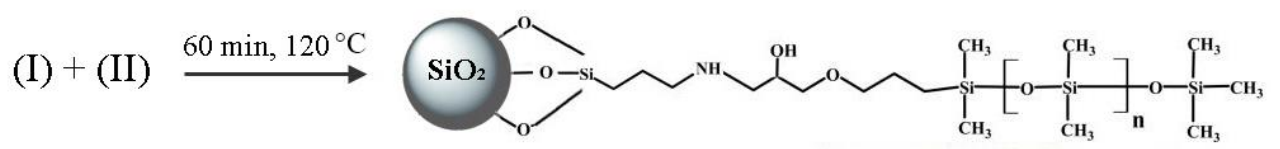

Scheme 1. Possible chemical reaction scheme for synthesis of amino-modified PDMS and superhydrophobic fabric. 


\subsection{Superhydrophobic property}

The hydrophobicity of fabrics was assessed using water contact angle (CA) measurement. The pristine fabric, due to the presence of hydrophilic hydroxyl and carboxyl groups, was completely wetted by water droplet. The in-situ deposition of silica nanoparticles makes the fabric surface rough, as depicted in SEM image (Figure 2b) and induces the hydrophobicity. After modification of silica-coated fabric with APTES-PDMS solution, the fabric was completely turned superhydrophobic (Figure 4), due to the low-surface energy of PDMS. The effect of PDMS concentration on hydrophobicity and contact angle of treated fabric was studied at $25{ }^{\circ} \mathrm{C}$ by changing it value in the range of 1 to $5 \%$. As can be seen on Figure 4, all the concentrations provide the superhydrophobicity, although the maximum contact angle $\left(155^{\circ}\right)$ was achieved at $2 \%$ concentration of PDMS. The high water contact angle obtained here is due to the formation of micro-nano roughness by silica nanoparticles as well as the low surface energy provided by PDMS. Table 1 summarized the hydrophobic properties of different substrate in term of fabrication approach, contact angle and washing fastness. As shown in Table 1, with respect to reported literature, the proposed fluorine-free coating formulation exhibited robust superhydrophobic with water contact angle higher than $150^{\circ}$.

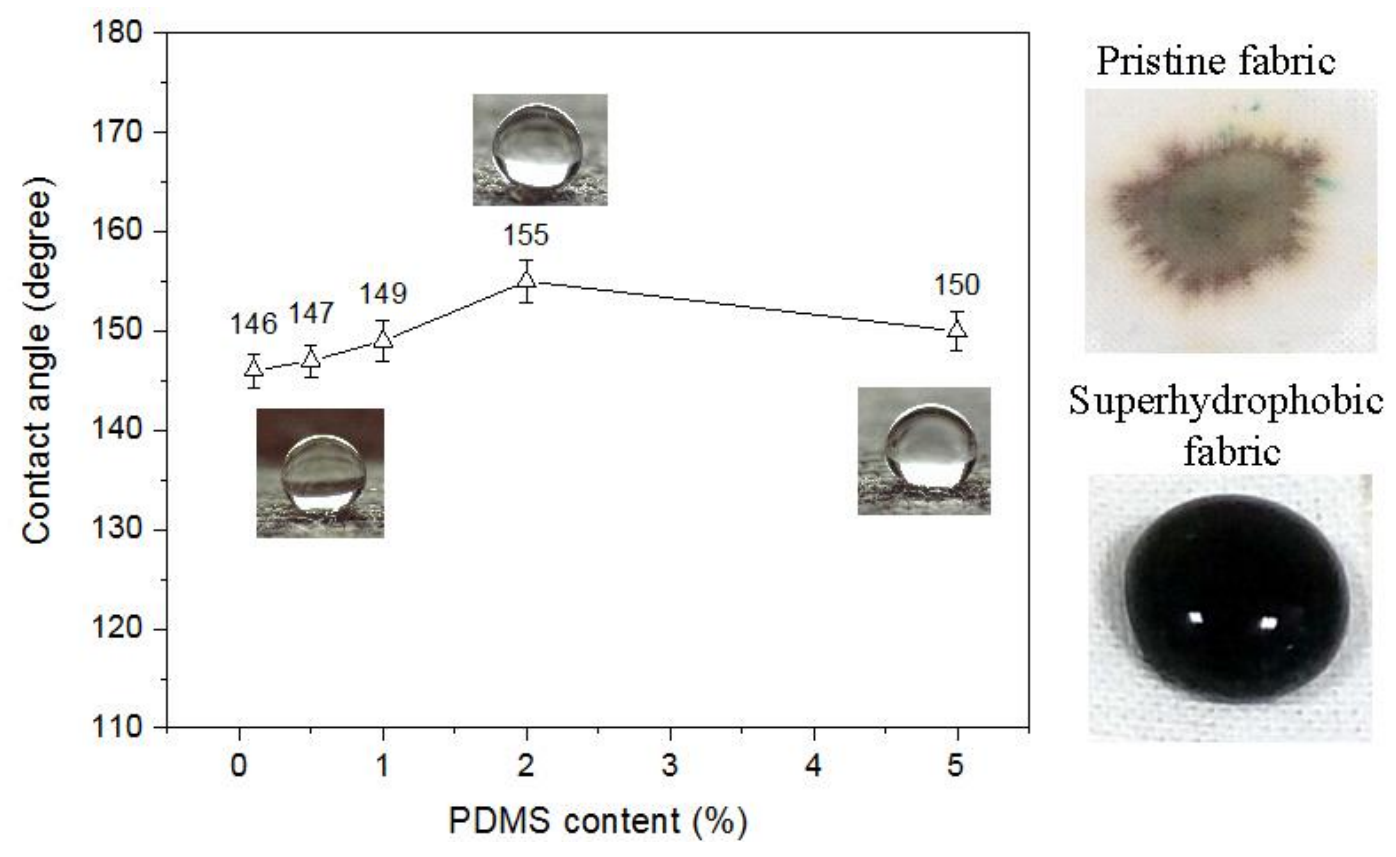


Figure 4. The effect of PDMS content on contact angle of treated fabrics.

Table 1. Comparison of hydrophobicity of different substrates using various approach

\begin{tabular}{|c|c|c|c|c|c|c|c|}
\hline No. & Substrate & Material & $\begin{array}{l}\text { Hydrophobic } \\
\text { agent }(\%)\end{array}$ & Method & $\begin{array}{l}\text { Contact } \\
\text { angle }\left(^{\circ}\right)\end{array}$ & $\begin{array}{c}\text { Wash } \\
\text { cycle } \\
\text { (number) }\end{array}$ & Ref. \\
\hline 1 & Cotton & $\begin{array}{l}\text { HDTMS- } \\
\text { GPTMS-TEOS }\end{array}$ & 3 & Immersion & 141 & 10 & [15] \\
\hline 2 & $\begin{array}{l}\text { Polyester- } \\
\text { cotton }\end{array}$ & $\begin{array}{l}\text { flour-acrylate } \\
\text { monomer }\end{array}$ & - & Plasma & 145 & - & [16] \\
\hline 3 & Silicon & Si with $\mathrm{XeF}_{2}$ & - & etch & 156 & - & {$[17]$} \\
\hline 4 & Cotton & $\begin{array}{l}\text { TEOS-OTES- } \\
\mathrm{AgNO}_{3}\end{array}$ & 3 & Immersion & 151 & - & [18] \\
\hline 5 & Cotton & $\begin{array}{l}\text { MTMS- } \\
\text { HDTMS }\end{array}$ & 3 & Sol-gel & 134 & - & [19] \\
\hline 6 & Cotton & MTCS & 1 & Immersion & 150 & - & [20] \\
\hline 7 & Silicon & $\begin{array}{l}\text { Modified } \\
\text { SiNPs with } \\
\text { APTS }\end{array}$ & 1 & Immersion & 143 & - & [21] \\
\hline 8 & Silicone & $\mathrm{PDMS} / \mathrm{SiO}_{2}$ & 4 & Immersion & 153 & 5 & [22] \\
\hline 9 & Glass & $\begin{array}{l}\text { PDMS- } \\
\text { nanosilica + } \\
\text { FAS }\end{array}$ & 4 & Spray & 158 & - & [23] \\
\hline 10 & Glass & $\begin{array}{l}\text { Polydimethylsi } \\
\text { loxane/silica }\end{array}$ & 0.6 & $\begin{array}{l}\text { Drop- } \\
\text { coating }\end{array}$ & 155 & - & [24] \\
\hline 11 & Cotton & TEOS-FAS & 1.4 & $\begin{array}{l}\text { Drop- } \\
\text { coating }\end{array}$ & 163 & 100 & [25] \\
\hline
\end{tabular}




\begin{tabular}{llllcccc}
\hline 12 & Glass & $\begin{array}{l}\text { PDMS and } \\
\text { polystyrene/ } \\
\mathrm{SiO}_{2}\end{array}$ & - & etch & 155 & 4 & {$[26]$} \\
13 & Glass & PDMS & 0.5 & Immersion & 120 & - & {$[27]$} \\
14 & Cotton & $\begin{array}{l}\text { PDMS- } \\
\text { ormosil }\end{array}$ & 2 & Immersion & 160 & 5 & {$[28]$} \\
15 & Polyester & $\begin{array}{l}\text { HDTMS - } \\
\text { TEOS }\end{array}$ & 4 & Immersion & 150 & 30 & {$[6]$} \\
16 & $\begin{array}{l}\text { Polyester- } \\
\text { viscose }\end{array}$ & $\begin{array}{l}\text { Modified silica } \\
\text { nanoparticles } \\
\text { with PDMS }\end{array}$ & 2 & Immersion & 152 & 25 & $\begin{array}{l}\text { This } \\
\text { study }\end{array}$ \\
\hline
\end{tabular}

\subsection{Physical properties}

The breathability and physical properties of pristine and superhydrophobic fabric were measured in terms of air permeability, crease recovery angle and bending length and the obtained results summarized at Table 2. The air permeability of treated fabrics, which serves as an indication of their breathability, was investigated. It can be seen that the superhydrophobic treatment of silicacoated fabric with PDMS-APTES solution drops moderately the air permeability of fabric about $36.3 \%$. Considering the crease recovery angle of fabrics (Table 2) indicate that the superhydrophobic fabric exhibits lower crease recovery angle than the pristine fabric. It is seen that for pristine fabric, the crease recovery angle in warp and weft directions are $155^{\circ}$ and $163^{\circ}$, respectively and both values gradually decreased by only $10.6 \%$ (warp) and $10.4 \%$ (weft) after superhydrophobic treatment. The effects of superhydrophobic treatment on bending rigidity of fabrics in both the warp and weft directions are also shown in Table 2. There were increases in bending length of superhydrophobic fabric for both the warp and weft directions. It could be attributed to the formation of hydrogen bonds between the hydroxyl group of fibers and the hydroxyl group of superhydrophobic coating, making the fiber a bit difficult to bend.

Table 2. Physical properties of pristine and superhydrophobic fabrics 


\begin{tabular}{|c|c|c|c|c|c|}
\hline \multirow{2}{*}{ Sample } & \multirow{2}{*}{$\begin{array}{c}\text { Air permeability } \\
\left(\mathrm{mL} / \mathrm{s} . \mathrm{cm}^{2}\right)\end{array}$} & \multicolumn{2}{|c|}{ Crease recovery angle $\left.{ }^{\circ}\right)$} & \multicolumn{2}{|c|}{ Bending length (cm) } \\
\cline { 3 - 6 } & 26.67 & warp & weft & warp & weft \\
\hline Pristine fabric & 17.00 & 138.5 & 146 & 2.94 & 1.61 \\
\hline $\begin{array}{c}\text { Superhydrophobic } \\
\text { fabric }\end{array}$ & & & 163 & 1.69 & 1.27 \\
\hline
\end{tabular}

\subsection{Durability}

To study the durability of treated fabric against laundering, they were subjected to several washing cycles. The CA of treated fabric as a function of washing cycles is shown in Figure 5. The obtained results imply that the $\mathrm{CA}$ of superhydrophobic fabric after 25 domestic washing cycles with water and $0.23 \%$ detergent solution decreased from $150^{\circ}$ to $143^{\circ}$.

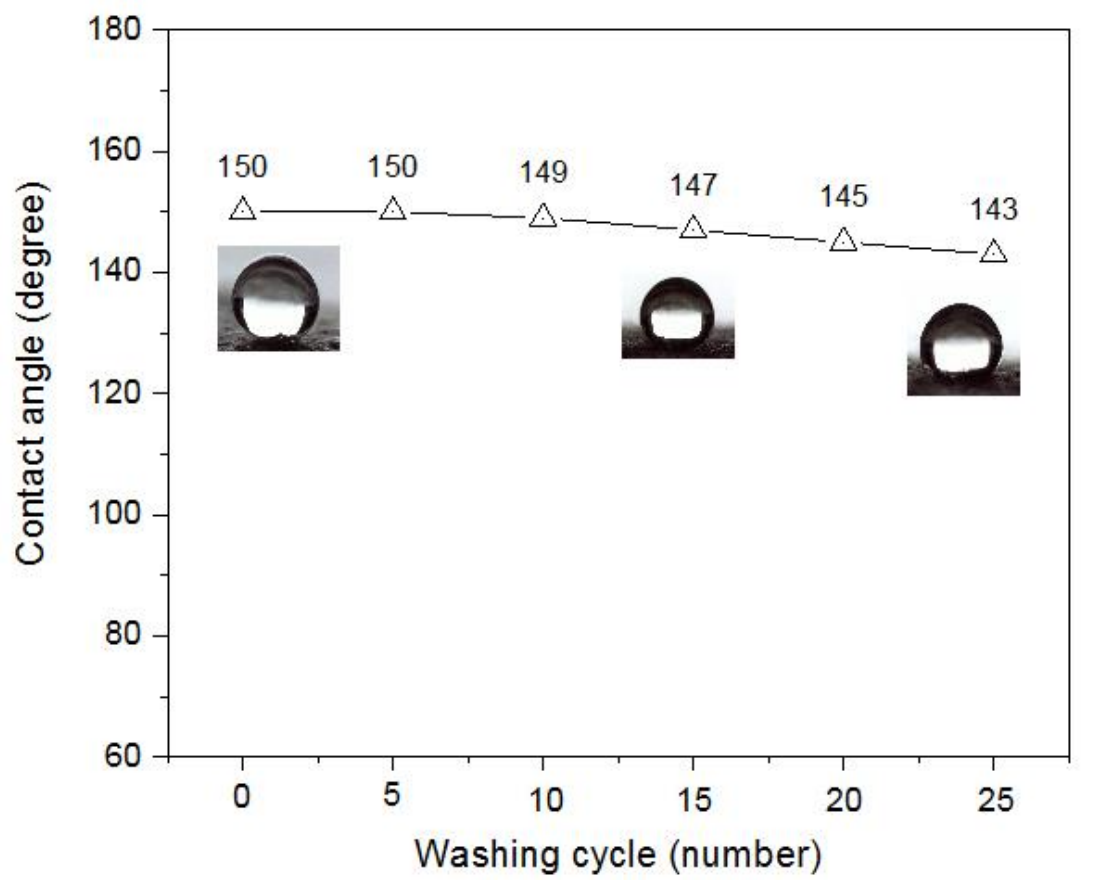

Figure 5. The contact angle of superhydrophobic fabrics as a function of washing cycles.

\section{Conclusion}

We have successfully fabricated superhydrophobic fabric by modifying the pristine fabric with silica nanoparticles followed by amino-modified PDMS through a dip coating process. The 
morphological study of treated fabric exhibits spherical silica nanoparticles distributed throughout the fabric surface, providing necessary nanoscale roughness for hydrophobicity. The prepared fabric shows outstanding superhydrophobicity with the contact angle of $151^{\circ}$ and long-term stability toward washing cycles. The employed compounds provided micro-nano roughness as well as the suitable functional groups favoring the low surface energy provided by PDMS. Considering the air permeability, bending length and crease recovery angle of superhydrophobic fabrics revealed that the treatment did not significantly affect the comfort properties of fabric.

Conflicts of Interest: The authors declare no conflict of interest.

\section{References}

[1] S. Asadollahi, J. Profili, M. Farzaneh, and L. Stafford, Development of Organosiliconbased superhydrophobic coatings through atmospheric pressure plasma polymerization of HMDSO in nitrogen plasma, Materials (Basel) 2019, 12, 2, 219.

[2] S. Liu, H. Zhou, H. Wang, Y. Zhao, H. Shao, Z. Xu, Z. Feng, D. Liu, T.S. Lin, Argon Plasma Treatment of Fluorine-Free Silane Coatings: A Facile, Environment-Friendly Method to Prepare Durable, Superhydrophobic Fabrics, Adv. Mater. Interfaces 2017, vol. 4, no. 11, 1-8.

[3] H. Liu, S.-W. Gao, J.-S. Cai, C.-L. He, J.-J. Mao, T.-X. Zhu, Z. Chen, J.-Y. Huang, K. Meng, K.-Q. Zhang, S.S. Al-Deyab and Y.-K. Lai, Recent Progress in Fabrication and Applications of Superhydrophobic Coating on Cellulose-Based Substrates, Materials (Basel) 2016, vol. 9, no. 3, 124.

[4] H. R. Hong, J. Kim, and C. H. Park, Facile fabrication of multifunctional fabrics: Use of copper and silver nanoparticles for antibacterial, superhydrophobic, conductive fabrics, RSC Adv. 2018, vol. 8, no. 73, 41782-41794.

[5] J. H. Oh, T. J. Ko, M. W. Moon, and C. H. Park, Nanostructured fabric with robust superhydrophobicity induced by a thermal hydrophobic ageing process, $R S C A d v . \mathbf{2 0 1 7}$, vol. 7, no. 41, 25597-25604.

[6] Q. Zhu, Q. Gao, Y. Guo, C. Q. Yang, and L. Shen, Modified Silica Sol Coatings for Highly Hydrophobic Cotton and Polyester Fabrics Using a One-Step Procedure, Ind. Eng. Chem. Res. 2011, pp. 5881-5888.

[7] H. F. Hoefnagels, D. Wu, G. De With, and W. Ming, Biomimetic Superhydrophobic and Highly Oleophobic Cotton Textiles, Langmuir 2007, no. 11, 13158-13163.

[8] B. Xu, Z. Cai, W. Wang, and F. Ge, Preparation of superhydrophobic cotton fabrics based on $\mathrm{SiO} 2$ nanoparticles and $\mathrm{ZnO}$ nanorod arrays with subsequent hydrophobic modification, Surf. Coat. Technol. 2010, vol. 204, no. 9-10, 1556-1561.

[9] C. H. Xue, M. Li, X. J. Guo, X. Li, Q. F. An, and S. T. Jia, Fabrication of superhydrophobic textiles with high water pressure resistance, Surf. Coatings Technol. 2017, vol. 310, 134-142.

[10] C. H. Xue, X. Bai, and S. T. Jia, Robust, Self-Healing Superhydrophobic Fabrics Prepared 
by One-Step Coating of PDMS and Octadecylamine, Sci. Rep. 2016, vol. 6, 1-11.

[11] M. Yang, W. Liu, C. Jiang, S. He, Y. Xie, and Z. Wang, Fabrication of superhydrophobic cotton fabric with fluorinated $\mathrm{TiO} 2$ sol by a green and one-step sol-gel process, Carbohydr. Polym. 2018, vol. 197, 75-82.

[12] P. Chauhan, A. Kumar, and B. Bhushan, Self-cleaning, stain-resistant and anti-bacterial superhydrophobic cotton fabric prepared by simple immersion technique, J. Colloid Interface Sci. 2019, vol. 535, 66-74.

[13] W. Stober, A. Fink, and D. Ernst Bohn, Controlled Growth of Monodisperse Silica Spheres in the Micron Size Range, J. Colloid Interface Sci. 1968, vol. 69, 62-69.

[14] P. Lampman, K. Vyvyan, D. L. Pavia, and G. S. Kriz, Introduction to spectroscopy. Cengage Learning, 2008.

[15] W. A. Daoud, J. H. Xin, and X. Tao, Superhydrophobic silica nanocomposite coating by a low-temperature process, J Am Ceram Soc 2004, vol. 87, no. 9, 1782-1784.

[16] S. A. Brewer and C. R. Willis, Structure and oil repellency App Surf Sci 2008, vol. 254, no. 20, 6450-6454.

[17] Y. Kwon, N. Patankar, J. Choi, and J. Lee, Design of Surface Hierarchy for Extreme Hydrophobicity Yongjoo, Langmuir 2009, vol. 25, no. 11, 6129-6136.

[18] M. Shateri Khalil-Abad and M. E. Yazdanshenas, Superhydrophobic antibacterial cotton textiles, J Colloid Interf Sci 2010, vol. 351, no. 1, 293-298.

[19] L. Xu, W. Zhuang, B. Xu, and Z. Cai, Fabrication of superhydrophobic cotton fabrics by silica hydrosol and hydrophobization, App Surf Sci 2011, vol. 257, no. 13, 5491-5498.

[20] M. A. Shirgholami, M. Shateri Khalil-Abad, R. Khajavi, and M. E. Yazdanshenas, Fabrication of superhydrophobic polymethylsilsesquioxane nanostructures on cotton textiles by a solution-immersion process, J Colloid Interf Sci 2011, vol. 359, no. 2, 530535.

[21] G. K. Raghuraman, C. Lu, Z. Zhang, and S. Yang, Highly Transparent and Superhydrophobic Surfaces from Co-assembly of Nanoparticles ( $\leq 100 \mathrm{~nm})$, Langmuir 2011, vol. 27, no. 7, 4594-4602.

[22] K. Li, X. Zeng, H. Li, X. Lai, C. Ye, and H. Xie, Study on the wetting behavior and theoretical models of polydimethylsiloxane/silica coating, App Surf Sci 2013, vol. 279, 458-463.

[23] B. J. Basu, V. Dinesh Kumar, and C. Anandan, Surface studies on superhydrophobic and oleophobic polydimethylsiloxane- silica nanocomposite coating system, App Surf Sci 2012, vol. 261, 807-814.

[24] Q. Ke, W. Fu, H. Jin, L. Zhang, T. Tang, and J. Zhang, Fabrication of mechanically robust superhydrophobic surfaces based on silica micro-nanoparticles and polydimethylsiloxane, Surf Coat Tech 2011, vol. 205, no. 21-22, 4910-4914.

[25] C. Zeng, H. Wang, H. Zhou, and T. Lin, Self-cleaning, superhydrophobic cotton fabrics with excellent washing durability, solvent resistance and chemical stability prepared from an SU-8 derived surface coating, $R S C A d v$. 2015, vol. 5, 610144-61050.

[26] C. H. Xue, Z. D. Zhang, J. Zhang, and S. T. Jia, Lasting and self-healing superhydrophobic surfaces by coating of polystyrene/ $\mathrm{SiO}_{2}$ nanoparticles and polydimethylsiloxane, J. Mater. Chem. A 2014, vol. 2, 15001-15007.

[27] J. Seo and L. P. Lee, Effects on wettability by surfactant accumulation/depletion in bulk polydimethylsiloxane (PDMS), Sensors Actuat B-Chem 2006, vol. 119, no. 1, 192-198.

[28] C. Cao, M. Ge, J. Huang, S. Li, S. Deng, S. Zhang, Z. Chen, K. Zhang, S.S. Al-Deyab, 
and Y. Lai, Robust fluorine-free superhydrophobic PDMS- ormosil@ fabrics for highly effective self-cleaning and efficient oil-water separation, J. Mater. Chem. A 2016, vol. 4, no. $31,12179-12187$. 